\title{
¿VIOLENCIA OBSTÉTRICA EN ESPAÑA, REALIDAD O MITO? 17.000 MUJERES OPINAN $^{1}$
}

\author{
Susana Iglesias ${ }^{1}$, Marta Conde ${ }^{2}$, Sofía González ${ }^{3}, \mathbf{M}^{\mathrm{a}}$ Esther Parada $^{4}$ \\ Autora correspondencia: Susana Iglesias Casás \\ Correo electrónico: matronasu@gmail.com
}

1. Matrona Hospital do Salnés (Vilagarcía de Arousa, Pontevedra).

2. Matrona Centro de Saúde de Muros, (Muros, A Coruña).

3. Matrona Hospital da Barbanza (Ribeira, A Coruña).

4. Matrona Hospital do Salnés (Vilagarcía de Arousa, Pontevedra).

Recibido: 28/12/2018 Aceptado: 21/01/2019

\footnotetext{
${ }^{1}$ Este trabajo ha sido presentado en forma de comunicación oral en el III Congreso Internacional de Género, Ética y Cuidado «Visibilizando la violencia hacia las mujeres en el embarazo», celebrado en Barcelona del 16 al18 de mayo de 2018.
} 


\section{RESUMEN}

\section{Objetivo}

Investigar la calidad percibida por las mujeres atendidas con motivo de un parto, cesárea o aborto en centros sanitarios españoles, y su satisfacción con distintos aspectos tanto humanos como técnicos de los cuidados recibidos.

\section{Material y Método}

Estudio descriptivo retrospectivo utilizando una encuesta online anónima difundida a través de redes sociales sobre la atención recibida durante su proceso obstétrico.

\section{Resultados}

Se obtuvo una muestra de 17.677 respuestas. La atención por parte de los sanitarios obtuvo una nota media de 6,9 sobre 10 . El 45,8 \% de las mujeres opinaron que los sanitarios no solicitaron su consentimiento informado antes de cada técnica realizada y el $49 \%$ que no tuvieron posibilidad de aclarar dudas o expresar miedos. El $38 \%$ percibieron que durante el parto recibieron procedimientos que no necesitaban y/o podían ser perjudiciales para su salud. El $34 \%$ de las mujeres opinaron que habían sufrido violencia obstétrica.

\section{Conclusiones}

El nacimiento de un nuevo hijo/a o su pérdida son eventos que dejan una importante huella en la salud física, psíquica y emocional de una mujer y de su familia. Los/as agentes de salud debemos reflexionar sobre el impacto generado por nuestros cuidados, la necesidad de basarlos en evidencia científica, y la obligación de respetar los derechos de la mujer y su bebé, aunque ni siquiera los conozca o los llegue a exigir. Los resultados de esta encuesta identifican una serie de áreas en las que pueden y deben mejorarse las prácticas de atención obstétrica, incluyendo el trato.

Palabras clave: derechos de las usuarias; relaciones profesional-paciente; violencia obstétrica. 


\section{ABSTRACT}

\section{Objective}

To investigate the quality of obstetric care as perceived by women in the context of giving birth, a cesarean section or miscarriage/stillbirth in health centers in Spain, and their satisfaction with different aspects of the attention received, both human and technical.

\section{Materials and method}

Retrospective descriptive study based on an anonymous online survey about the care received during the obstetric process.

\section{Results}

A sample of 17,677 answers was obtained. Healthcare professionals were awarded an average of 6.9 points out of $10.45 .8 \%$ of women believed that healthcare professionals did not ask for their informed consent before every procedure and $49 \%$ did not have the opportunity to clarify doubts or express fears. $38 \%$ perceived that they received unnecessary or potentially dangerous procedures during labor. Finally, $34 \%$ believed they had suffered obstetric violence.

\section{Conclusions}

The birth or loss of a baby are events that leave a deep mark on the physical, psychological and emotional health of women and their families. Healthcare professionals should reflect upon the impact of our care, the need to base it on scientific evidence and the duty to respect women's and children's rights, even if they do not know these rights nor demand them. The results of this survey contribute to drawing up a guideline and identifying a series of fields in which obstetric attention, including treatment, can and must be improved.

Keywords: patient rights; professional-patient relationship; obstetric violence. 


\section{INTRODUCCIÓN}

En la Ley Orgánica sobre el Derecho de las Mujeres a una Vida Libre de Violencia, publicada en Venezuela en 2007, se define la violencia obstétrica (VO) como la «apropiación del cuerpo y procesos reproductivos de las mujeres por personal de salud, que se expresa en un trato jerárquico deshumanizador, en un abuso de medicalización y patologización de los procesos naturales, trayendo consigo pérdida de autonomía y capacidad de decidir libremente sobre sus cuerpos y sexualidad, impactando negativamente en la calidad de vida de las mujeres» (Venezuela, 2007).

A nivel global, el tema es lo suficientemente importante como para generar declaraciones y manifiestos de distintas organizaciones e instituciones (Asamblea General ONU, 1993; White Ribbon Alliance, 2011), e incluso la Organización Mundial de la Salud afirmó que «El maltrato, la negligencia o la falta de respeto en el parto pueden constituir una violación de los derechos humanos fundamentales de las mujeres, descritos en las normas y los principios internacionales de derechos humanos» y que «Todas las mujeres tienen derecho a recibir en el embarazo y en el parto el más alto nivel de cuidados en salud, que incluye el derecho a una atención digna y respetuosa, y el derecho a no sufrir violencia ni discriminación» (OMS, 2014). Estaríamos ante otra forma de violencia de género cuyas repercusiones pueden ser graves y afectar a la salud física y emocional del binomio madre-bebé a corto y largo plazo (Olza, 2013; Belli, 2013; Bergman y Bergman, 2013; Souza, Rattner y Gubert, 2017; McGarry, 2017).

Según indica Bellón Sánchez en 2015 «Desde 2007 varios países latinoamericanos (México, Argentina, y Venezuela) han adoptado leyes en contra de la VO. Poco a poco, este término ha sido también utilizado por movimientos sociales en diversas partes del mundo para denunciar lo que muchas mujeres entienden como prácticas irrespetuosas y violentas ejercidas por el personal sanitario durante la atención de sus embarazos, partos y lactancias».

Aunque durante décadas haya sido una forma de violencia negada, invisibilizada e incluso normalizada, en los últimos años se ha despertado interés público por el tema en España. El hecho de que en nuestro país no exista por el momento una ley específica sobre VO no quiere decir que un profesional sanitario no pueda incurrir en responsabilidades legales al llevar a cabo ciertas prácticas o actitudes durante el parto que vulneren los derechos fundamentales de la mujer. 
La calidad en la atención obstétrica en nuestro país es deficitaria a niveles alarmantes, tanto desde el punto de vista científico o biomédico, como desde el respeto a la dignidad, privacidad y a la autonomía de las mujeres. El Informe sobre la Atención al Parto y Nacimiento en el Sistema Nacional de Salud, publicado por el Ministerio de Sanidad en el año 2012 (MSSSI), nos ofrece datos claros sobre ciertos indicadores de calidad cuyo cumplimiento está muy lejos de los estándares establecidos: tasas excesivas de episiotomías en partos normales, de cesáreas, o de partos normales atendidos en posición de litotomía, entre otros. A veces, la falta de cumplimiento de las recomendaciones y la VO se justifican por parte de los sanitarios/as en base a la falta de tiempo, el estrés, la sobrecarga asistencial, la falta de medios materiales e infraestructuras o el ejercicio de una medicina defensiva basada en el temor a un potencial proceso de judicialización (Goberna y Boladeras, 2018). También se defiende frecuentemente la teoría de «casos puntuales», según la cual sería muy poco habitual que una mujer sufriera VO en un hospital español. Otros sanitarios van más allá y niegan incluso la mera existencia de este grave problema de salud pública, o la justifican en aras de haber priorizado proteger la salud del neonato (Carvalho, 2012; Pintado, Penagos y Casas, 2015).

Todo esto nos lleva a afirmar que, probablemente, tanto los sanitarios como el resto de la sociedad han normalizado cierta violencia como inherente al proceso de convertirse en madre. Muchos ciudadanos/as consideran que el elevado número de intervenciones a las que se ven sometidas las mujeres durante su parto son necesarias e incuestionables, y desconocen sus consecuencias a corto y largo plazo sobre su salud y la de sus hijos/as. Quizás una excesiva confianza en la tecnología y el desconocimiento sobre la fisiología del parto favorece que este tipo de violencia permanezca enmascarada.

El caso es que las mujeres no se quejan o apenas lo hacen: para algunos profesionales y gestores, es fácil deducir entonces que están satisfechas con todo o la mayor parte de lo sucedido en su proceso. Pero también puede ser que esa parálisis venga derivada de la anulación, indefensión, culpa e impotencia que genera la violencia.

El Ministerio de Sanidad recomienda realizar encuestas a nivel autonómico dirigidas a las mujeres para conocer su grado de satisfacción con la atención recibida en el parto (MSSSI, 2012), aceptando que es necesario investigar para conocer la herramienta y el momento más adecuado para obtener esta valiosa información para que sirva de evaluación de profesionales y mejora de los servicios. 
Este trabajo responde a una necesidad personal y profesional de «objetivar» la existencia de VO en España. Nuestro objetivo es conocer la calidad percibida por las mujeres atendidas con motivo de un parto, cesárea o aborto en centros sanitarios españoles y su satisfacción con distintos aspectos tanto humanos como técnicos de los cuidados recibidos (apoyo emocional, provisión de información, uso de lenguaje, autonomía en la toma de decisiones y pertinencia de los procedimientos obstétricos). Además, deseamos explorar qué porcentaje de mujeres considera haber padecido VO durante su proceso, así como investigar su satisfacción global con la atención recibida.

\section{MATERIAL Y MÉTODO}

\section{Diseño}

Estudio observacional descriptivo.

\section{Población}

Mujeres atendidas con motivo de un parto, cesárea o aborto en un hospital público o privado español en los 9 años anteriores al momento de rellenar el cuestionario. En 2007 fue publicada la Estrategia de la Atención al Parto Normal (Ministerio de Sanidad y Consumo, 2007), y a partir de ese momento sus recomendaciones deberían haber sido consideradas en toda institución sanitaria.

\section{Criterios de exclusión}

Partos atendidos en casa o en hospitales fuera del territorio español.

\section{Instrumento de recogida de información}

Cuestionario anónimo, voluntario y externo al sistema sanitario. Al no encontrar una herramienta validada para la evaluación de la VO, el equipo investigador desarrolló un cuestionario propio tras la revisión de la bibliografía consultada sobre el tema (Asociación Civil Dando a Luz, 2010; Terán et al., 2013; Rodríguez y Aguilera, 2017). Utilizamos la plataforma online «Google Forms», que permitía el uso de la lógica de saltos entre preguntas.

La introducción del cuestionario explicaba el propósito del estudio y su naturaleza anónima. Para no sesgar la obtención de los datos, en la encuesta no aparecía el término 
Violencia Obstétrica hasta el final. Utilizamos preguntas cerradas para facilitar el manejo y el análisis de los datos. Incluimos una sola pregunta abierta (la última), donde las mujeres podían volcar sus sugerencias de mejora en atención perinatal. Todas las preguntas eran de obligada respuesta, de lo contrario no podía avanzarse en el cuestionario ni enviar los resultados a la plataforma.

Se realizó una prueba piloto durante 1 semana, donde se envió el cuestionario a 100 madres para que aportasen comentarios, sugerencias de cambios de redacción (y así asegurar que la interpretación de la pregunta coincidía con su intención), y eliminación o adición de elementos.

\section{Variables}

En la encuesta se incluyeron 16 ítems que agrupamos en 7 bloques. (Ver Tabla n. 1) En las preguntas de los bloques 3, 4,5 y 6 se les preguntó además la categoría del profesional sanitario que las atendió (matrona, pediatra, ginecólogo, etc.) y el nivel de atención donde fueron atendidas (atención primaria/especializada).

Tabla 1. Bloques e ítems de la encuesta

\begin{tabular}{|l|l|}
\hline \multicolumn{1}{|c|}{ Bloques } & \multicolumn{1}{c|}{ Ítems } \\
\hline 1. Apoyo institucional & $\begin{array}{l}\text { Las instituciones (Ministerio de Sanidad, Servicios de } \\
\text { Salud, Gerencias) apoyan eficazmente y promueven } \\
\text { derechos en el embarazo, parto y puerperio/lactancia: } \\
\text { sí/no/no sabe-no contesta) }\end{array}$ \\
\hline $\begin{array}{l}\text { 2. Valoración global de la } \\
\text { atención recibida }\end{array}$ & $\begin{array}{l}\text { Satisfacción global con la atención recibida por parte de } \\
\text { los profesionales sanitarios: Escala 1-10, donde 1 es la } \\
\text { peor puntuación posible y 10 la máxima. }\end{array}$ \\
\hline $\begin{array}{l}\text { 3. Aspectos específicos de } \\
\text { la atención recibida por } \\
\text { los profesionales sanitarios } \\
\text { en el proceso } \\
\text { embarazo/parto/puerperio }\end{array}$ & $\begin{array}{l}\text { Sensación/estado de ánimo tras la atención sanitaria } \\
\text { recibida: empoderada y satisfecha/culpable e } \\
\text { insegura/indiferente/no sabe-no contesta }\end{array}$ \\
\hline $\begin{array}{l}\text { Obtención del consentimiento informado antes de cada } \\
\text { uno de los procedimientos y técnicas que te iban a realizar, } \\
\text { explicando las opciones que tenías: sí/no/no sabe-no } \\
\text { contesta. }\end{array}$ \\
$\begin{array}{l}\text { Verbalización de comentarios irónicos o descalificadores: } \\
\text { sí/no/no sabe-no contesta. }\end{array}$ \\
$\begin{array}{l}\text { Uso de sobrenombres o diminutivos infantilizadores para } \\
\text { dirigirse a ti: sí/no/no sabe-no contesta. }\end{array}$
\end{tabular}




\begin{tabular}{|c|c|}
\hline & $\begin{array}{l}\text { - Posibilidad de aclarar dudas o expresar miedos o } \\
\text { inquietudes: sí/no/no sabe-no contesta. } \\
\text { - Información sobre existencia del plan de parto: sí/no/no } \\
\text { sabe-no contesta. } \\
\text { - Posibilidad aclaración dudas sobre plan de parto: sí/no/no } \\
\text { sabe-no contesta. }\end{array}$ \\
\hline $\begin{array}{l}\text { 4. Cuidados recibidos en el } \\
\text { aborto/muerte perinatal }\end{array}$ & $\begin{array}{l}\text { - Provisión de apoyo psicológico o emocional: sí/no/no } \\
\text { sabe-no contesta. } \\
\text { - Proporción de cuidados que consideras innecesarios o } \\
\text { injustificados: sí/ no/ no sabe-no contesta. }\end{array}$ \\
\hline $\begin{array}{l}\text { 5. Cuidados recibidos en el } \\
\text { parto/cesárea }\end{array}$ & $\begin{array}{l}\text { - Respeto del plan de parto presentado: sí/ no/ no sabe-no } \\
\text { contesta. } \\
\text { - Explicación de por qué no se respetó plan de parto } \\
\text { presentado: sí/no/no sabe-no contesta. } \\
\text { - Recepción de cuidados que consideras innecesarios y/o } \\
\text { dañinos: sí/no/no sabe-no contesta. } \\
\text { - Intervenciones que consideras se te realizaron } \\
\text { innecesariamente: listado cerrado de } 20 \text { procedimientos } \\
\text { perinatales habituales. }\end{array}$ \\
\hline $\begin{array}{l}\text { 6. Atención y apoyo } \\
\text { recibido por los } \\
\text { profesionales sanitarios } \\
\text { durante la lactancia } \\
\text { materna/crianza }\end{array}$ & $\begin{array}{l}\text { - Apoyo a decisiones sobre la alimentación y cuidado de tu } \\
\text { bebé: sí/no/no sabe-no contesta. } \\
\text { - Apoyo eficaz y ayuda para solventar dudas o dificultades } \\
\text { surgidas con la lactancia materna: sí/no/no sabe-no } \\
\text { contesta. }\end{array}$ \\
\hline $\begin{array}{l}\text { 7. Percepción violencia } \\
\text { obstétrica }\end{array}$ & $\begin{array}{l}\text { - Consideras haber sufrido violencia obstétrica durante la } \\
\text { atención de tu proceso: sí/no/no sabe/no contesta. }\end{array}$ \\
\hline
\end{tabular}

\section{Captación y reclutamiento de las participantes}

Las mujeres que participaron lo hicieron voluntariamente. No se les pidió ningún dato de carácter personal mediante el cual se las pudiese identificar.

Se implementó un muestreo no probabilístico a través de la técnica de «bola de nieve», utilizada en otros estudios que han abordado la calidad de la atención sanitaria perinatal (Rodríguez y Aguilera, 2017; Redondo et al., 2005; Mira et al., 2012).

Difundimos el formulario a través de profesionales sanitarios, asociaciones de crianza, grupos de apoyo a la lactancia, administradoras de blogs, la asociación «El Parto es Nuestro», y enviando el enlace a la encuesta a través de redes sociales como WhatsApp o Facebook. La recogida de datos duró tres meses (de febrero a abril de 2018) 


\section{Análisis de los datos}

Una vez concluido el trabajo de campo, se procedió al tratamiento de los datos mediante el programa SPSS en su versión 19.

\section{RESULTADOS}

Se obtuvieron 17.742 encuestas rellenadas. Se descartaron 65 por proceder de mujeres que dieron a luz en el extranjero, obteniéndose 17.677 respuestas válidas.

\section{Apoyo institucional (Gerencias, Servicios de Salud, Ministerio de Sanidad)}

El 60,6\% $(\mathrm{n}=10.720)$ de las encuestadas opinaron que no se apoyan eficazmente o promueven lo suficiente sus derechos en embarazo, parto o puerperio/lactancia.

\section{Valoración global de la atención recibida por parte de los sanitarios}

La atención por parte de los sanitarios obtuvo una nota media de 6,9 medida en una escala del 1(menor satisfacción) al 10 (mayor satisfacción). Otros datos sobre la valoración global de la atención recibida por los sanitarios pueden consultarse en la Tabla 2.

Tabla 2 Valoración global de la atención recibida por sanitarios en el proceso obstétrico

\begin{tabular}{|c|c|}
\hline Valoración & \\
\hline Nota media & 6,9 \\
\hline \multicolumn{2}{|l|}{ Atención recibida hizo sentir } \\
\hline Empoderada y satisfecha & $6.832(41,5 \%)$ \\
\hline Insegura, vulnerable, culpable & $6.491(39 \%)$ \\
\hline No sabe/no contesta & $4.354(19,5 \%)$ \\
\hline
\end{tabular}

\section{Valoración de aspectos específicos de la atención recibida por parte de los sanitarios:}

El 39,1\% de las mujeres $(n=6.924)$ afirman que los sanitarios no les informaron sobre su derecho a entregar un plan de parto, y el 12,3\% $(n=2.194)$ que no les resolvieron dudas sobre el mismo. Pueden consultarse otros aspectos específicos sobre la atención del proceso obstétrico en la Tabla 3. 
Tabla 3. Actitudes del personal sanitario durante la atención del proceso obstétrico

\begin{tabular}{|c|c|c|c|c|}
\hline Actitud & $\%(\mathbf{n})$ & $\begin{array}{c}\text { Categoría } \\
\text { profesional } \\
\text { sanitario n }(\%)\end{array}$ & $\begin{array}{l}\text { Momento del } \\
\text { proceso n (\%) }\end{array}$ & $\begin{array}{c}\text { Nivel de } \\
\text { atención n (\%) }\end{array}$ \\
\hline $\begin{array}{c}\text { NO solicitud del } \\
\text { consentimiento informado } \\
\text { antes de cada técnica o } \\
\text { procedimiento. }\end{array}$ & $8.093(45,8)$ & $\begin{array}{c}\text { Ginecólogo/a: } \\
5.958(73,6) \\
\text { Matrona: } \\
4.173(51,5) \\
\text { Enfermera/o: } \\
2.399(29,6) \\
\text { Anestesista: } \\
1.266(15,6)\end{array}$ & \begin{tabular}{l}
\multicolumn{1}{c}{ Parto: } \\
6.512 $(80,4)$ \\
Embarazo: \\
3.065 $(37,8)$ \\
Puerperio: \\
$1.658(20,4)$
\end{tabular} & $\begin{array}{c}\text { Especializada: } \\
7.614(94) \\
\text { Primaria: } \\
1.493(18,4)\end{array}$ \\
\hline $\begin{array}{l}\text { Verbalización de críticas al } \\
\text { comportamiento de la } \\
\text { mujer, o descalificaciones. }\end{array}$ & $6.076(34,3)$ & $\begin{array}{c}\text { Ginecólogo: } \\
2.661(43,8) \\
\text { Matrona: } \\
2.615(43) \\
\text { Enfermera/o: } \\
2.115(34,8) \\
\text { TCAE: } \\
\text { 1.080 }(17,7)\end{array}$ & $\begin{array}{c}\text { Parto: } \\
4.137(68) \\
\text { Embarazo: } \\
\text { 1.876 (31) } \\
\text { Puerperio: } \\
1.798(29,5)\end{array}$ & $\begin{array}{l}\text { Especializada: } \\
5.608(92,2) \\
\text { Primaria: } \\
1.141(18,7)\end{array}$ \\
\hline $\begin{array}{c}\text { Uso de } \\
\text { sobrenombres/diminutivos } \\
\text { infantilizadores. }\end{array}$ & $5.530(31,2)$ & $\begin{array}{c}\text { Matrona: } \\
2.715(49) \\
\text { Enfermera/o: } \\
2.456(44,4) \\
\text { Ginecólogo/a: } \\
\text { 1.449 }(26,2) \\
\text { TCAE: } \\
\text { 1.684 }(30,4)\end{array}$ & $\begin{array}{l}\text { Parto: } \\
4087(74) \\
\text { Puerperio: } \\
1.690(30,5) \\
\text { Embarazo: } \\
1.563(28,2)\end{array}$ & $\begin{array}{l}\text { Especializada: } \\
5.396(97,5) \\
\text { Primaria: } \\
1.580(28,1)\end{array}$ \\
\hline $\begin{array}{l}\text { NO posibilidad de aclarar } \\
\text { dudas, o expresar miedos o } \\
\text { inquietudes }\end{array}$ & 8.673 (49) & $\begin{array}{c}\text { Matrona: } \\
3.875(44,6) \\
\text { Enfermera/o: } \\
2.682(31) \\
\text { Ginecólogo/a: } \\
5.290(61) \\
\text { Pediatra: } \\
1.065(12,3)\end{array}$ & $\begin{array}{c}\text { Embarazo: } \\
3.111(36) \\
\text { Parto: } \\
\text { 5.568 }(64,1) \\
\text { Puerperio: } \\
2.818(32,4)\end{array}$ & $\begin{array}{c}\text { Especializada: } \\
7.693(88,7) \\
\text { Primaria: } \\
1.910(22)\end{array}$ \\
\hline $\begin{array}{c}\text { Plan de parto: } \\
\text { *Información sobre su } \\
\text { existencia } \\
\text { * Posibilidad aclaración de } \\
\text { dudas }\end{array}$ & $\begin{array}{l}10.818(60,9) \\
15.548(87,6)\end{array}$ & & & \\
\hline
\end{tabular}

TCAE: Técnico en Cuidados Auxiliares de Enfermería 


\section{Percepción calidad de cuidados recibidos en caso de aborto/muerte perinatal}

De las mujeres que contestaron el cuestionario, 1.428 (8\%) sufrieron un aborto o muerte perinatal. En un $70 \%$ de los casos estas mujeres percibieron una falta del apoyo emocional que requiere una pérdida o proceso de duelo.

Tabla 4: Percepción atención sanitaria en el aborto/muerte perinatal

\begin{tabular}{|l|c|}
\hline \multicolumn{1}{|c|}{ A tención sanitaria } & n (\%) \\
\hline Práctica de cuidados innecesarios/injustificados & $338(23,7)$ \\
\hline Falta de apoyo emocional & $1.011(70,8)$ \\
\hline
\end{tabular}

\section{Percepción calidad de cuidados recibidos en caso de parto/cesárea:}

El 50,1\% (n=8.870) de las mujeres encuestadas no llegaron a entregar plan de parto. El 44,2 \% ( $n=3.896$ ) de las mujeres que sí lo entregaron opinan que no se respetó. Entre los profesionales que consideran que no respetaron su plan de parto tenemos a los ginecólogos con un 59,8 \%, a las matronas con un 54,2 \% y las enfermeras con un $20,8 \%$ (podían escoger varias categorías). A estas mujeres cuyo plan de parto no se respetó, en un $55,1 \%$ de los casos $(n=2.149)$ tampoco se les explicaron los motivos para no hacerlo.

El $38 \%$ de las mujeres opinaron que recibieron procedimientos que no necesitaban y/o podían ser potencialmente perjudiciales para su salud. Los profesionales más implicados en estas técnicas percibidas como innecesarias fueron los ginecólogos en un $78 \%$ de los casos, y en el $77 \%$ las matronas (podían seleccionar más de un profesional implicado). Entre las técnicas o situaciones con más frecuencia percibidas por las mujeres como injustificadas o innecesarias destacan usar oxitocina artificial $(64,2 \%$; $n=4.321)$ la falta de información sobre el proceso $(55,7 \% ; n=3.749)$, impedirles moverse libremente $(52,3 \% ; n=3.521)$, práctica de la episiotomía $(52,3 \% ; n=3.517)$, separarlas de su bebé sano (49,3\%; $\mathrm{n}=3.317)$, realizar amniotomía $(45,5 \%$; $=3.257)$, y realizar la maniobra de Kristeller (45,5\%, n=3.064) (podían seleccionar más de una práctica). El resto de los procedimientos que las mujeres consideraron que se les realizó de manera injustificada y/o dañinos pueden consultarse en la Tabla 4. El 60,1\% $(n=4.042)$ de las mujeres que recibieron estos cuidados percibidos como innecesarios afirman que no se le explicaron razones de estos procedimientos ni se le pidió consentimiento previamente. 
Tabla 5. Percepción atención sanitaria intraparto/cesárea

\begin{tabular}{|c|c|}
\hline Percepción & n $(\%)$ \\
\hline Recibiste procedimientos que consideras innecesarios y/o dañinos & $6.723(38)$ \\
\hline Ginecólogo/a & $5.239(78)$ \\
\hline Matrona & $5.172(77)$ \\
\hline Enfermera & $2.882(43)$ \\
\hline Pediatra & $1.219(18)$ \\
\hline $\begin{array}{l}\text { No te explicaron razones de estos procedimientos innecesarios ni te } \\
\text { pidieron consentimiento }\end{array}$ & $4.042(60,1)$ \\
\hline No se respetó tu plan de parto & $3.896(44,2)$ \\
\hline Profesional no lo respetó & \\
\hline Ginecólogo/a & $2.330(59,8)$ \\
\hline Matrona & $2.114(54,2)$ \\
\hline Enfermera/o & $813(20,8)$ \\
\hline Pediatra & $253(6,5)$ \\
\hline No te explicaron motivos de no haber respetado tu plan de parto & $2.149(55,1)$ \\
\hline Procedimientos que consideras recibiste innecesariamente: & $4.321(64,2)$ \\
\hline Uso de oxitocina artificial & $3.749(55,7)$ \\
\hline No aportar información sobre el proceso & $3.521(52,3)$ \\
\hline Impedir mover libremente & $3.517(52,3)$ \\
\hline Episiotomía & $3.317(49,3)$ \\
\hline Separarte del bebé sano & $3.257(48,4)$ \\
\hline Rotura artificial bolsa amniótica & $3.064(45,5)$ \\
\hline Maniobra Kristeller & $3.058(45,4)$ \\
\hline Impedir comer o beber & $2.900(43,1)$ \\
\hline Llevarse bebé para realizarle técnicas (analíticas, etc.) & $2.854(42,4)$ \\
\hline Tactos vaginales muy seguidos y por distintas personas & $2.508(37,3)$ \\
\hline Impedir estar acompañada & $2.134(31,7)$ \\
\hline Ventosa/fórceps & $1.921(28,5)$ \\
\hline Maniobra Hamilton & $1.890(28,1)$ \\
\hline Pinzamiento precoz cordón umbilical & $1.524(22,6)$ \\
\hline Cesárea & $1.230(18,2)$ \\
\hline
\end{tabular}




\begin{tabular}{|r|r|}
\hline Administrar al RN biberones sin consentimiento & $1.017(15,1)$ \\
Extracción manual placenta & $814(12,1)$ \\
Enema & $695(10,3)$ \\
Rasurado pubis & $2.194(32,6)$ \\
Otro/s procedimiento/s & \\
\hline
\end{tabular}

\section{Percepción calidad de cuidados recibidos durante lactancia materna/crianza:}

El 36,3\% de las mujeres ( $n=5.779)$ no se sintió apoyada en sus decisiones sobre aspectos relacionados con el cuidado o la alimentación de su recién nacido. De entre las mujeres que optaron por amamantar a su bebé, el 40,2 \% (n=6.160) sintieron escaso apoyo a la lactancia materna por parte de los sanitarios cuando esta presentó alguna dificultad.

Tabla 6. Percepción atención sanitaria durante la lactancia materna/crianza

\begin{tabular}{|c|c|c|c|c|}
\hline Actitud & n $(\%)$ & $\begin{array}{l}\text { Catego } \\
\text { sanitar }\end{array}$ & $\begin{array}{l}\text { orofesional } \\
\text { nplicado } \mathbf{n} \\
\text { b) }\end{array}$ & $\begin{array}{c}\text { Nivel atención } \\
\text { n (\%) }\end{array}$ \\
\hline \multirow{4}{*}{$\begin{array}{c}\text { Falta de apoyo en } \\
\text { decisiones sobre } \\
\text { alimentación/cuidado del } \\
\text { bebé }\end{array}$} & \multirow{4}{*}{$5.779(36,3)$} & \multicolumn{2}{|c|}{ Enfermera/o: $3.458(59,8)$} & Primaria: \\
\hline & & Pediatra: & $2.127(36,8)$ & $2.381(41,2)$ \\
\hline & & TCAE: & $1.654(28,6)$ & Especializada: \\
\hline & & Matrona: & $1.296(22,4)$ & $4.079(70,5)$ \\
\hline \multirow{4}{*}{$\begin{array}{l}\text { Falta de apoyo y solución } \\
\text { de problemas en caso de } \\
\text { lactancia materna }\end{array}$} & \multirow{4}{*}{$6.160(40,2)$} & \multicolumn{2}{|c|}{ Enfermera/o: $4.140(67,2)$} & Primaria: \\
\hline & & Pediatra: & $2.355(38,2)$ & $2.692(43,7)$ \\
\hline & & Matrona & $2.113(34,3)$ & Especializada: \\
\hline & & & & $4.456(72,4)$ \\
\hline
\end{tabular}

TCAE: Técnico en Cuidados Auxiliares de Enfermería

\section{Percepción de violencia obstétrica:}

El 34, 3\% de las mujeres encuestadas (6.077) considera haber sufrido violencia obstétrica.

\section{DISCUSIÓN}

Durante la prueba piloto de nuestro cuestionario, muchas madres nos comentaron que necesitaban más preguntas abiertas para poder expresar todas sus inquietudes con respecto a la atención recibida. Por motivos de diseño de estudio, esto no fue posible. De todas maneras, deseamos plantear su reivindicación para futuras investigaciones sobre el 
tema. Muchas participantes nos expresaron su alegría al haber encontrado una vía (aunque sea tan pobre como un cuestionario) para comunicar sus sentimientos, frustraciones o satisfacción con respecto a la atención recibida durante su proceso.

Consideramos que una de las fortalezas del estudio es la naturaleza anónima de la encuesta online, que facilita que las participantes revelen detalles sobre temas socialmente estigmatizados o tabú como el que nos ocupa. Pensamos que el gran número de mujeres a las que hemos podido llegar al haberse virilizado el cuestionario en redes sociales no hubiese sido posible alcanzarlo con ningún otro método (entrevista telefónica o encuesta con soporte papel) en tan corto plazo de tiempo (tres meses).

En cuanto a las limitaciones de este estudio, está el utilizar un cuestionario propio al no haber encontrado una encuesta validada para la evaluación de la VO. No se calculó el Alfa de Cronbach para validar su consistencia interna. Además, entendemos que los resultados no son extrapolables a la población general de mujeres que han dado a luz en España entre 2009 y 2018, ya que no todas tuvieron la misma oportunidad de responder a la encuesta. Probablemente la muestra presente sesgos importantes en el perfil sociodemográfico, y hayan respondido menos mujeres pertenecientes a estratos de población marginados (etnias minoritarias, bajo nivel de ingresos, inmigrantes, nivel de educación formal bajo) sin acceso a internet y, por lo tanto, sin posibilidad de participar. Según la bibliografía consultada (Perera et al., 2018; Fonn y Xaba, 2001; Cáceres y Nieves, 2017), es posible que la VO se practique más sobre esas mujeres, por lo que la VO percibida real sería de mayor magnitud que la estimada en nuestros resultados.

Otro posible sesgo de la muestra podría deberse a haber utilizado, entre otros medios de difusión, a asociaciones que reivindican los derechos en la atención al parto y en el nacimiento. De esta manera, podría haber llegado con más probabilidad a mujeres que consideran que han padecido VO y se acercan a estos grupos en busca de apoyo emocional. Aun así, observamos que sólo el $50 \%$ de las mujeres encuestadas han entregado un plan de parto durante su proceso, porcentaje similar al de otros estudios publicados sobre el tema (Vázquez Gutiérrez, 2013). Por lo tanto, creemos que la muestra es bastante representativa del nivel de conocimiento sobre los derechos en embarazo, parto y puerperio de la población española en general.

En cuanto al posible sesgo de memoria que pudiese afectar a las mujeres participantes, el análisis se limitó a casos de un máximo de 9 años previos a la encuesta 
y parece un margen más que aceptable para poder recordar fielmente un acontecimiento tan importante como el nacimiento de un hijo/a (Sinkin, 1992; Lundgren, Karlsdottir y Bondas, 2009; Yawn, Suman y Jacobsen, 1998).

El 34,3\% de mujeres que participaron en esta encuesta afirmaron haber percibido VO por parte de los profesionales sanitarios durante su proceso. Este porcentaje es semejante al de otros trabajos similares publicados (Da Silva y Santana, 2017; Wilde et al., 2011; Brandao, 2018).

A través de los resultados de nuestra encuesta, encontramos que la VO no solo ocurre en el parto (aunque es cierto que en esta etapa se manifiesta en mayor medida), sino también en el embarazo y puerperio. Asimismo, que no es una práctica que afecte exclusivamente a matronas y ginecólogos, sino que las mujeres también identifican a otros actores sanitarios, como anestesistas, pediatras, enfermeras y auxiliares de enfermería.

Aproximadamente el $40 \%$ de las mujeres encuestadas opinaron que recibieron cuidados que consideran innecesarios. En algunas ocasiones, esta percepción de las mujeres podría deberse a un problema de confianza o entendimiento con los profesionales que la atendían. En ese caso, entendemos que es necesario un esfuerzo en la mejora de la relación profesional-usuaria basada en el respeto, la información veraz y el acompañamiento emocional. En otras ocasiones, los procedimientos descritos por las mujeres se ha demostrado científicamente que son perjudiciales y deben ser evitados, como el enema, el rasurado del pubis o la maniobra de Kristeller. Al realizarlos, se contraviene la evidencia científica y las recomendaciones sanitarias internacionales publicadas por la Organización Mundial de la Salud (WHO, 1985) y las del Ministerio de Sanidad reflejadas en la Estrategia de Atención al Parto normal (Ministerio de Sanidad y Consumo, 2007). Además, el $60 \%$ de estas mujeres afirmaron que estos cuidados, para ellas improcedentes, se les realizaron incluso sin pedirles consentimiento previo, y por lo tanto violando la Ley 41/2002, de 14 de noviembre, Básica reguladora de la Autonomía del Paciente y de Derechos y Obligaciones en materia de Información y Documentación Clínica (España, 2002).

De la revisión de buenas prácticas referidas por la literatura nacional e internacional (OMS, 2014; MSSSI, 2012; WHO, 1985; España, 2002), se destaca la importancia de promover la participación informada de las mujeres en todo el proceso y en la toma de 
decisiones sobre las prácticas clínicas que se le apliquen, posiblemente uno de los aspectos detectados como más deficitarios en nuestra encuesta. En ella el 45,8 \% (8.093 mujeres) afirmaron que no se les pidió consentimiento informado para realizarles distintas técnicas o procedimientos habituales en la atención obstétrica. Creemos que esto se debe al paternalismo sanitario que fomenta la dependencia del usuario/a y cuestiona a las pocas personas que eligen tomar decisiones sobre su salud. En base a nuestra experiencia profesional, pensamos que en general se valora muy positivamente la «obediencia» del paciente y se critica a los que se salen de esta norma, al entenderse esto como una puesta en duda de la autoridad sanitaria. Más que ofrecer técnicas o tratamientos, estos se imponen sin presentar alternativas (Goberna y Boladeras, 2018), y se confunde la preferencia del sanitario con la única verdad o posibilidad existente, aunque a veces esté fundada en bases tan cuestionables como la falta de actualización, la tradición, o sus inseguridades y prejuicios.

Desde que la mayoría de los nacimientos en los países desarrollados se dan en el ámbito hospitalario, ha dominado el modelo médico y hegemónico que considera el parto una «situación de riesgo». Así, muchos profesionales validan exponer a la mujer y al recién nacido a una medicalización del proceso que conlleva iatrogenia pero que a ellos posiblemente les proporciona una sensación falsa de «control» sobre el mismo (Biurrun Garrido, 2017; Coxon, Sandall and Fulop, 2013). El hecho de que cada vez haya más generaciones de mujeres que no han vivido su parto de manera fisiológica, y más promociones de profesionales que no han tenido la oportunidad de presenciar nacimientos ocurridos en tales circunstancias, retroalimenta y perpetúa la idea del parto como experiencia atemorizante. Todo esto hace tan necesario como difícil un cambio de paradigma de una obstetricia con un enfoque biomecanicista y medicalizado y fundamentado en el riesgo a otro basado en la salutogénesis, en la preservación de la fisiología, y en la salud (Meier Magistretti et al., 2016; Ferguson et al., 2016).

Un pilar básico para conseguir mejorar este aspecto es que la matrona sea el profesional que lidere un modelo de atención continuada a las mujeres sanas en embarazo, parto, puerperio y lactancia. Se ha demostrado que este modelo de cuidado disminuye la posibilidad de que necesiten intervenciones, aumenta su satisfacción sin aumentar la mortalidad ni morbilidad, y es costoefectivo (Sandall et al., 2016). Todas las intervenciones y tecnología, así como la participación de los ginecólogos/as en el proceso deben quedar reservadas exclusivamente para escasas usuarias que sí los necesitan, y no 
deben ofrecerse preventivamente a todas las mujeres, ya que, según la evidencia científica, su uso generalizado no evita complicaciones, sino que las causa.

A la luz de los resultados de esta encuesta, y a pesar del tiempo transcurrido desde la publicación de la EAPN (Ministerio de Sanidad, 2007), continúa existiendo la necesidad de aportar una atención sanitaria en el proceso obstétrico más centrada en las necesidades particulares de las mujeres, que garantice sus derechos, elimine prácticas innecesarias y/o nocivas y entienda por fin el parto como un proceso fisiológico en el que en el $85 \%$ de los casos la mujer no necesita más que un acompañamiento experto y respetuoso (WHO, $1985 ; 2000)$.

Los resultados de este estudio manifiestan que, aunque ciertos sanitarios y sociedades científicas nieguen la existencia de la VO (Sociedad Española de Ginecología y Obstetrícia, 2018), o la consideren en ocasiones «necesaria» para asegurar el bienestar maternofetal, no son pocas las mujeres que perciben que la atención sanitaria que han recibido es deficitaria en calidad y rigor científico, e identifican numerosos aspectos de mejora de la asistencia al proceso obstétrico tanto desde el punto de vista tecnológico como humano. Y estas opiniones expresadas por las mujeres coinciden con los datos del Informe sobre la Atención al Parto y Nacimiento en el Sistema Nacional de Salud, publicado por el Ministerio de Sanidad, Servicios Sociales e Igualdad en el año 2012 de manera indiscutible. 


\section{BIBLIOGRAFÍA}

Asamblea General de las Naciones Unidas. Declaration on the Elimination of Violence against Women [en línea]. Asamblea General de las Naciones Unidas, 1993. Disponible en: 〈www.refworld.org/docid/3b00f25d2c.html $>$.

Asociación Civil Dando a Luz. Test de violencia obstétrica [en línea]. Buenos Aires: Asociación Civil Dando a Luz. Disponible en:

<http://dandoaluz.org.ar/pdfs/TestdeViolenciaObstetrica.pdf $>$.

BELLI, L. La violencia obstétrica: otra forma de violación a los derechos humanos. En: Revista Redbioética. 2013, vol. 1, núm. 7, pp. 25-34.

BELLÓN SÁNCHEZ, S. La violencia obstétrica desde los aportes de la crítica feminista y la biopolítica. En: Dilemata. 2015, vol. 18, pp. 93-111.

Bergman, J.; Bergman, N. Whose Choice? Advocating Birthing Practices According to Baby’s Biological Needs. En: Journal of Perinatal Education. 2013, vol. 22, núm. 1, pp. 8-13.

BIURRUn GARRIDO, A. La humanización de la asistencia al parto: Valoración de la satisfacción, autonomía y del autocontrol [tesis doctoral]. Barcelona: Dipòsit Digital de la Universitat de Barcelona, 2017. Disponible en:

$<$ http://hdl.handle.net/2445/118125>.

BRANDÃO, T. et al. Childbirth experiences related to obstetric violence in public health units in Quito, Ecuador. En: Int J Gynaecol Obstet. 2018, vol. 143, núm. 1, pp. 84-8.

CÁceres, F. M.; NiEves, G. M. Humanized care in childbirth. Difference according to maternal clinical and social conditions. En: Rev Colomb Obstet Ginecol. 2017, vol. 68, pp. $128-34$

CARvalho, V. F. et al. How the workers of a birthing center justify using harmful practices in natural childbirth. En: Rev. Esc. Enferm. 2012, vol. 46, núm. 1, pp. 2935.

Coxon, K.; Sandall, J.; Fulop, N. J. To what extent are women free to choose where to give birth? How discourses of risk, blame and responsibility influence birth place decisions. En: Health, Risk \& Society. 2013, vol. 16, núm. 1, pp. 51-67.

DA SILVA, I.; SANTANA, R. Formas de violencia obstétrica experimentada por madres que tuvieron un parto normal. En: Enferm. glob. 2017, vol. 16, núm. 4, pp. 71-97. 
ESPAÑA. Ley 41/2002, de 14 de noviembre, Básica reguladora de la Autonomía del Paciente y de Derechos y Obligaciones en materia de Información y Documentación Clínica. Boletín Oficial del Estado. 15 de noviembre de 2002, núm. 274, pp. 40126-32

FERGUSON, S. et al. Sense of coherence and women's birthing outcomes: A longitudinal survey. En: Midwifery. 2016, vol. 34, pp. 158-165. Disponible en:

<www.ncbi.nlm.nih.gov/pubmed/26689770>.

FonN, S.; XABA, M. Health workers for change: developing the initiative. En: Health Policy Plan. 2001, vol. 16, pp. 13-8.

GOBERnA, J.; BoladerAs, M. (coords.). El concepto «Violencia obstétrica» y el debate actual sobre la atención al nacimiento. Madrid: Tecnos, 2018.

LUNDGREN, I.; KARLSDOTTIR, S. I.; BONDAS, T. Long-term memories and experiences of childbirth in a Nordic context - a secondary analysis. En: Int J Qual Studies Health Well-being. 2009, vol. 4, núm. 2, pp. 115-28.

MCGARRY, J. et al. Experiences and impact of mistreatment and obstetric violence on women during childbearing: a systematic review protocol. En: JBI Database System Rev Implement Rep. 2017, vol. 3, pp. 620-7.

Meier Magistretti, C. et al. Setting the stage for health: Salutogenesis in midwifery professional knowledge in three European countries. En: Int J Qual Stud Health Wellbeing. 2016, vol. 11, núm. 1.

Ministerio de SAnidad y Consumo. Estrategia de Atención al Parto Normal en el Sistema Nacional de Salud [en línea]. Madrid: Ministerio de Sanidad y Consumo, 2007. Disponible en: <www.elpartoesnuestro.es/>.

MiNISTERIO DE SANIDAD, SERVICIOS SOCIALES E IGUALDAD. Informe sobre la Atención al Parto y Nacimiento en el Sistema Nacional de Salud [en línea]. Madrid: MSSSI, 2012. Disponible en: 〈www.mscbs.gob.es〉.

MiRA, J. J. et al. Calidad de la atención al paciente oncológico. Tiempos asistenciales recomendables entre sospecha clínica y definición del plan terapéutico en cáncer de mama y colorrectal. Anales Sis San Navarra. 2012;35(3):385-393

OLZA, I. PTSD and obstetric violence. En: Midwifery Today with International Midwife. 2013, vol. 105, pp. 48-9, 68.

ORganización Mundial de la SALud. Prevención y erradicación de la falta de respeto y el maltrato durante la atención del parto en centros de salud [en línea]. OMS, 2014. Disponible en:

<www.who.int/reproductivehealth>. 
PERERA, D. et al. When helpers hurt: women's and midwives' stories of obstetric violence in state health institutions, Colombo district, Sri Lanka. En: BMC Pregnancy Childbirth. 2018, vol. 18, núm. 1, pp. 211.

Pintado, S.; Penagos, J. C.; CASAS, M. A. Síndrome de desgaste profesional en médicos y percepción de la violencia obstétrica. En: Ginecol Obstet Mex. 2015, vol. 83, núm. 3, pp. 173-8.

REDONDO, S. et al. Percepciones y expectativas sobre la atención primaria de salud: una nueva forma de identificar mejoras en el sistema de atención. En: Aten Primaria. 2005, vol. 36, núm. 7, pp. 358-66.

Rodríguez, P. A.; AguiLERA, L. La violencia obstétrica, otra forma de violencia contra la mujer. El caso de Tenerife. En: Musas. 2017, vol. 2, núm. 2, pp. 56-74

SANDALL, J. et al. Midwife-led continuity models versus other models of care for childbearing women. En: Cochrane Database of Systematic Reviews. 2016, vol. 4.

SIMKIN, P. Just another day in a woman's life? Part II: Nature and consistency of women's long-term memories of their first birth experiences. En: Birth. 1992, vol. 19, núm. 2, pp. 64-81.

SOCIEDAD ESPAÑOLA DE GINECOLOGÍA Y OBSTETRICIA. Violencia obstétrica: Un concepto legalmente delictivo, moralmente inadecuado, científicamente inaceptable [en línea]. Madrid: Sociedad española de Ginecología y obstetricia; 2018. Disponible en: 〈https://us18.campaign-archive.com>.

SOUZA, K. J.; RATTNER, D.; GUBERT, M. B. Institutional violence and quality of service in obstetrics are associated with postpartum depression. En: Revista Saude Publica. 2017, vol. 51, pp. 69.

TERÁn, P. et al. Violencia obstétrica: percepción de las usuarias. En: Rev Obstet Ginecol Venez. 2013, vol. 73, núm. 3, pp. 171-80.

VÁZQUeZ GuTIÉRREZ, B. El plan de parto. Una herramienta para la visibilización de las mujeres [trabajo final de máster]. Castellón de la Plana: Publicaciones de la Universitat Jaume I, 2013.

VENEZUELA. Ley Orgánica sobre el derecho de las mujeres a una vida libre de violencia. Gaceta Oficial. 2007, núm. 38.668.

White RibBon Alliance. Respectful Maternity Care: The Universal Rights of Childbearing Women [en línea]. Washington DC: White Ribbon Alliance, 2011. Disponible en: <http://whiteribbonalliance.org $>$. 
WHO - World Health Organization. Appropriate technology for birth. En: Lancet. 1985, vol. 326, pp. 436-7.

WHO-World Health Organization. Manejo de las complicaciones del embarazo y el parto: guía para obstetrices y médicos. Ginebra: OMS, 2000. Disponible en: 〈www.who.int/maternal_child_adolescent/documents/9241545879/es/>.

WILDE, B. et al. Birthgiving women's feelings and perceptions of quality of intrapartal care: a nationwide Swedish cross-sectional study. En: J Clin Nurs. 2011, vol. 20, núm. 7-8, pp. 1168-77.

YAWN, B. P.; SUMAN, V. J.; JACOBSEN S. J. Maternal recall of distant pregnancy events. En: J Clin Epidemiol. 1998, vol. 51, núm. 5, pp. 399-405. 